\title{
Voltage Stabilization in MVDC Microgrids Using Passivity-Based Nonlinear Control*
}

\author{
Andrea Martinelli, Pulkit Nahata, Giancarlo Ferrari-Trecate
}

\begin{abstract}
This paper investigates the application of passivity-based nonlinear control to the problem of primary voltage stabilization in medium-voltage DC microgrids (MVDC mGs) given by the interconnection of nonlinear distributed generation units (DGUs) and power lines. To this aim, we propose nonlinear local regulators which steer the voltage at the output terminal of each DGU to a reference value. Each controller can be explicitly synthesized relying on DGU parameters, voltage reference values of the neighboring DGUs and resistance of the neighboring power lines. The control design enables plug-and-play (PnP) operations: a plug-in or -out of a DGU requires only the update of regulators of neighboring DGUs without spoiling the stability of overall $\mathrm{mG}$. Theoretical results are backed up by simulations in Simulink environment.
\end{abstract}

\section{INTRODUCTION}

An $\mathrm{mG}$ is a group of spatially-distributed systems composed of loads and distributed generation units (DGUs), interconnected to each other through an electrical network [9], [12]. The advantages of a distributed energy infrastructure with respect to the classical centralized paradigm are numerous. They include the capability of electrifying remote areas, islands, or large buildings, the ability of improving resilience to faults and power quality in power networks, and the possibility of operating in both grid connected and islanded modes [9], [14]. These attractive features of mGs make them a promising operational architecture for future power systems. Motivated by advances in power electronics, batteries and renewable DC energy sources, DC mGs, nowadays, find applications in various field such as highefficiency households, electric vehicles, hybrid energy storage systems, data centres, avionics and marine systems [6], [14]. Moreover, as control of reactive power or unbalanced electric signals is not an issue, the control and management of a DC system is much simpler than in $\mathrm{AC}$, which makes DC mGs practically more feasible.

A key problem in islanded DC mGs is to ensure voltage stability through decentralized control architectures at the primary level of each DGU [5], [14]. Popular solutions for mGs in low-voltage (LV) configuration are based on droop controllers, but their stabilization properties have been shown either for specific $\mathrm{mG}$ topologies, or for general

\footnotetext{
*This work has received support from the Swiss National Science Foundation under the COFLEX project (grant number 200021_169906).

A. Martinelli is with Dipartimento di Elettronica, Informazione e Bioingegneria, Politecnico di Milano, Italy. He has moved to the Automatic control Laboratory at ETH Zürich, Switzerland. Email: andrea5.martinelli@mail.polimi.it

P. Nahata and G. Ferrari-Trecate are with Automatic Control Laboratory, École Polytechnique Fédérale de Lausanne (EPFL), Switzerland. Email: \{pulkit.nahata, giancarlo.ferraritrecate\}@epfl.ch

Corresponding author: P. Nahata
}

topologies but relying on networked secondary regulators [9], [20]. An alternative class of controllers for LV mGs, called Plug-and-Play ( $\mathrm{PnP}$ ), have been proposed, amongst others, in [19] and [18]. The main feature of PnP approach is that, independent of $\mathrm{mG}$ size and topology, the addition or removal of DGUs does not require the retuning of all the other local regulators to preserve voltage stability. Recently, the necessity of interconnecting remote power networks, such as off-shore wind farms, is pushing towards the introduction of higher voltage DC grids [8]. Different approaches have been proposed for the primary control of both $\mathrm{AC}$ [1] and DC [21], [7] higher voltage mGs. In [21] nonlinear droop control techniques are investigated, while in [7], after an impulse-response-based grid modelling, an MPC hierarchical architecture is provided.

The main target of this paper is the application of passivity-based tools for studying voltage stability in MVDC mGs. Passivity theory is a powerful framework to analyse complex systems, both with linear and non-linear dynamics [10]. It permits to design control actions based on system's energetic considerations, and has strong relationship with Lyapunov stability [11]. Moreover, when dealing with largescale systems, passivity theory provides a compositional framework. In fact, the interconnection of passive dynamical systems can present useful stability properties, depending on the nature of the communication channels and on the topology of the network [4], [17], [3].

Contributions: In this work, we show that the class of local controllers proposed in [16] passivate MV DGUs, composed of a DC renewable source and a nonliear DC/DC Boost converter. Relying on the results developed in [17] for passive subsystems interconnected in a skew-symmetric fashion, we characterize the asymptotic behaviour of the $\mathrm{mG}$ states and prove global asymptotic stability of the entire $\mathrm{mG}$. The proposed control architecture is decentralized and the synthesis of local regulators can be performed explicitly without utilizing optimization-based tools. Furthermore, the control design facilitates addition or removal of nodes in a PnP fashion.

Paper organization: In the next Section, we introduce the electrical model of an MVDC mG, composed by DGUs and power lines. After a global equilibria analysis, performed in Section III, the decentralized nonlinear control architecture is presented in Section IV. Section V is devoted to prove passivity of closed-loop DGUs and power lines. In Section VI, we show that the passivity of individual DGUs results in global asymptotic stability of the network. In the last section, we validate our control architecture through simulations. 


\section{Preliminaries and notation}

Notation: We let $\mathbb{R}$ denote the set of real numbers. Given $x \in \mathbb{R}^{n}, \operatorname{diag}(x) \in \mathbb{R}^{n \times n}$ is the associated diagonal matrix with elements of $x$ on the diagonal. We denote by $\mathcal{G}(\mathcal{V}, \mathcal{E})$ a digraph, where $\mathcal{V}=\{1, \cdots, N\}$ is the node set and $\mathcal{E} \subseteq$ $(\mathcal{V} \times \mathcal{V})$ is the edge set. For node $i \in \mathcal{V}, \mathcal{N}_{i}^{+}=\{j \in \mathcal{V}$ : $(i, j) \in \mathcal{E}\}$ denotes the set of out-neighbors, $\mathcal{N}_{i}^{-}=\{j \in \mathcal{V}$ : $(j, i) \in \mathcal{E}\}$ the set of in-neighbors, and $\mathcal{N}_{i}=\mathcal{N}_{i}^{+} \cup \mathcal{N}_{i}^{-}$the set of neighbors.

Passivity theory: Consider a control-affine nonlinear system

$$
\Sigma_{N L}:\left\{\begin{array}{l}
\dot{x}=q(x, u)=f(x)+g(x) u \\
y=h(x)
\end{array},\right.
$$

where $x \in \mathbb{R}^{n}, y \in \mathbb{R}^{p}$, and $u \in \mathbb{R}^{p}$. The functions $q$ : $\mathbb{R}^{n} \times \mathbb{R}^{p} \rightarrow \mathbb{R}^{n}, f: \mathbb{R}^{n} \rightarrow \mathbb{R}^{n}, g: \mathbb{R}^{n} \rightarrow \mathbb{R}^{n} \times \mathbb{R}^{p}$, and $h: \mathbb{R}^{n} \rightarrow \mathbb{R}^{p}$ are twice continuously differentiable, verifying $q(0,0)=0, f(0)=0$, and $h(0)=0$. Note that inputs and outputs have the same dimension $p$.

Definition 1: The nonlinear system $\Sigma_{N L}$ is passive [10] if there exists a continuously differentiable positive-semidefinte storage function $V: \mathbb{R}^{n} \rightarrow \mathbb{R}, V(x) \geq 0, V(0)=0$, and a function $\psi: \mathbb{R}^{n} \rightarrow \mathbb{R}, \psi(x) \geq 0 \forall t \geq 0$, such that

$$
\dot{V}(x) \leq u^{T} y-\psi(x) .
$$

The system $\Sigma_{N L}$ is strictly passive if $x \neq 0 \Rightarrow \psi(x)>0$.

\section{MiCROGRID MODEL}

The MV $\mathrm{mG}$ is composed of two different classes of dynamical systems, namely DGUs and lines. Their electric interconnections are modeled using a directed, connected, bipartite graph $\mathcal{G}=(\mathcal{V}, \mathcal{E})$. The set of nodes $\mathcal{V}=\{1, \ldots, N\}$ is consequently partitioned into two subsets, $\mathcal{S}_{A}$ and $\mathcal{S}_{L}$, such that any edge $(i, j) \in \mathcal{E}$ connects only nodes of different subsets. Sets $\mathcal{S}_{A}$ and $\mathcal{S}_{L}$ respectively collect $p$ DGUs and $q$ lines, such that $p+q=N$. We refer the reader to Figure 1 for a representative network diagram. The orientation of each edge represents the reference direction of positive currents which is arbitrarily assigned. It is evident that a line cannot have only in-neighbors or out-neighbors as the current entering in a line must leave it. Indeed, each node in $\mathcal{S}_{L}$ is always connected to two different nodes in $\mathcal{S}_{A}$ through two directed edges. We, therefore, describe the interaction network with the following $p \times q$ incidence matrix

$$
B=\left[b_{i j}\right], \quad b_{i j}=\left\{\begin{aligned}
1 & \text { if } j \in \mathcal{N}_{i}^{+} \\
-1 & \text { if } j \in \mathcal{N}_{i}^{-} \\
0 & \text { otherwise }
\end{aligned}\right.
$$

where $i \in \mathcal{S}_{A}$ and $j \in \mathcal{S}_{L}$.

Dynamic model of a power line: The power line $j$ is modeled as an $R L$ circuit with resistance $R_{L j}>0$ and inductance $L_{L j}>0$. It connects two DGUs, which in Figure $2 \mathrm{~b}$ are denoted with $x$ and $y$. By applying Krichoff's voltage law (KVL) on the $j^{\text {th }}$ power-line, one obtains

$$
\Sigma_{[j]}^{\text {line }}:\left\{L_{L j} \dot{I}_{L j}=V_{n e t, j}-R_{L j} I_{L j} \quad, j \in \mathcal{S}_{L},\right.
$$

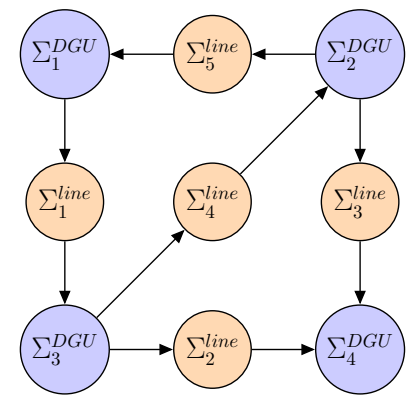

Fig. 1: A representative graph of an $\mathrm{mG}$ with four DGUs.

where $I_{L j}$ is the inductor current. The term

$$
V_{n e t, j}=\sum_{k \in \mathcal{N}_{j}} b_{k j} V_{k}
$$

is the sum of PCC voltages of the neighbours $k \in \mathcal{N}_{j}$ of line $j$ and accounts for the coupling of lines with the rest of the network.

Dynamic model of a DGU: Each DGU comprises a DC voltage source (usually generated by a renewable resource) and a Boost converter, which steps up the input voltage $V_{i n, i}$ to obtain an output voltage $V_{i}$ such that $V_{i}>V_{i n, i}$. The $i^{t h}$ DGU feeds a local load at the $i^{\text {th }}$ point of common coupling (PCC) and is connected to other DGUs through power lines. A schematic electric diagram of the a DGU along with load is represented in Figure 2a. The Boost converter is equipped with a high-frequency switch $D_{i}$ that enables transitions into two possible discrete states: "on" and "off" with the switch being respectively open and close. It is assumed that the Boost converter always remains in continuous conduction mode, that is, the inductor current $I_{i}$ does not fall to zero at any point in time. This enables one to write an averaged dynamic model [15] as

$$
\Sigma_{[i]}^{D G U}:\left\{\begin{array}{l}
C_{i} \dot{V}_{i}=d_{i} I_{i}-I_{\text {load }, i}-I_{\text {net }, i} \\
L_{i} \dot{I}_{i}=-d_{i} V_{i}-R_{i} I_{i}+V_{\text {in }, i}
\end{array} \quad, i \in \mathcal{S}_{A}\right.
$$

where $I_{n e t, i}$, a function of neighbouring line currents, is the net current injected into the $\mathrm{mG}$. It is given by

$$
I_{n e t, i}=\sum_{j \in \mathcal{N}_{i}} b_{i j} I_{L j}
$$

and represents the coupling of the DGU with the rest of the mG. In (6), $V_{i}, V_{i n, i}, I_{i}$, and $I_{l o a d, i}$ are the PCC voltage, input voltage, inductor current, and load current, respectively. The variable $d_{i} \in(0,1)$ is called the duty cycle of the converter and is an input variable. Notice that DGUs (6) are non-linear, since $d_{i}$ multiplies variables $V_{i}$ and $I_{i}$.

\section{STUdy OF THE EQUilibRIA}

The lines (4) and DGUs (6), linked together with linear couplings (5) and (7), represent the MV network. In this section, we study the existence and multiplicity of the equilibria of the network. 


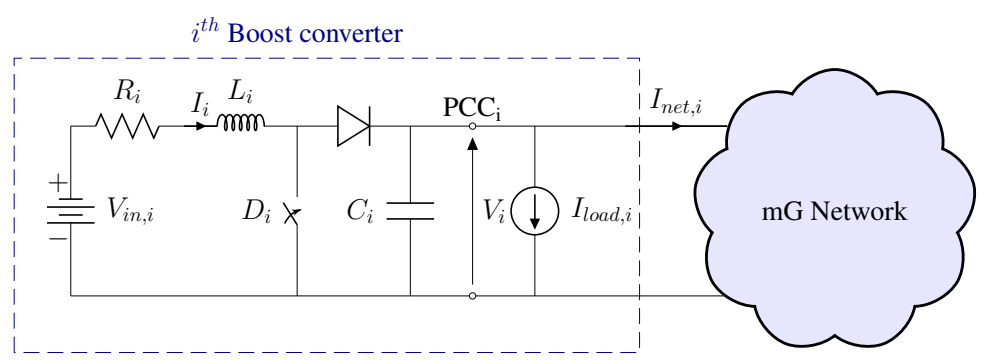

(a) $i^{t h}$ Boost converter connected to the $\mathrm{mG}$

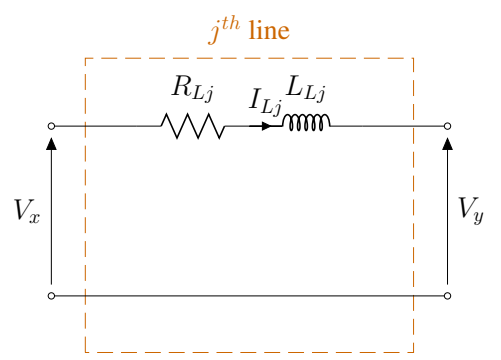

(b) $j^{\text {th }}$ line connecting a couple of DGUs

Fig. 2: The MV mG is composed of two classes of dynamical systems, namely DGUs (a) and lines (b).

\section{A. Equilibrium existence and uniqueness}

We define the the global state of the network as

$$
\mathbf{x}=\left[\begin{array}{c}
V \\
I \\
I_{L}
\end{array}\right] \in \mathbb{R}^{(2 p+q) \times 1},
$$

where $V=\left[V_{1}, \cdots, V_{p}\right]^{T}, I=\left[I_{1}, \cdots, I_{p}\right]^{T}$, and $I_{L}=$ $\left[I_{L 1}, \cdots, I_{L q}\right]^{T}$. On considering constant exogenous terms $I_{l o a d, i}$ and $V_{i n, i}$, and constant duty cycles $d_{i} \in(0,1) \forall i \in$ $\mathcal{S}_{A}$, one can write (6) and (4) in a compact matrix form as

$$
\dot{\mathbf{x}}=A \mathbf{x}+Q .
$$

Matrices $A \in \mathbb{R}^{(2 p+q) \times(2 p+q)}$ and $Q \in \mathbb{R}^{p \times 1}$ are

$A=\left[\begin{array}{cc|c}\mathbf{0}_{p \times p} & C^{-1} D & -C^{-1} B \\ -L^{-1} D & -L^{-1} R & \mathbf{0}_{p \times q} \\ \hline L_{L}^{-1} B^{T} & \mathbf{0}_{q \times p} & -L_{L}^{-1} R_{L}\end{array}\right], Q=\left[\begin{array}{c}-C^{-1} I_{l d} \\ L^{-1} V_{i n} \\ \mathbf{0}_{q \times 1}\end{array}\right]$

where $C=\operatorname{diag}\left(C_{1}, \cdots, C_{p}\right), L=\operatorname{diag}\left(L_{1}, \cdots, L_{p}\right)$, $R=\operatorname{diag}\left(R_{1}, \cdots, R_{p}\right), R_{L}=\operatorname{diag}\left(R_{L 1}, \cdots, R_{L q}\right), L_{L}=$ $\operatorname{diag}\left(L_{L 1}, \cdots, L_{L q}\right), I_{l d}=\left[I_{\text {load }, 1}, \cdots, I_{\text {load }, p}\right]^{T}, V_{\text {in }}=$ $\left[V_{i n, 1}, \cdots, V_{i n, p}\right]^{T}, D=\operatorname{diag}\left(d_{1}, \cdots, d_{p}\right)$ and $B$ is the incidence matrix defined in (3).

Lemma 1 (Equilibrium existence and uniqueness): The equilibrium of system (9), when matrices $A$ and $Q$ are constant, exists and is unique.

Proof: Let $\overline{\mathrm{x}}$ be an equilibrium state of system (9). Hence,

$$
A \overline{\mathrm{x}}=-Q .
$$

The uniqueness of the equilibrium hinges on proving the invertibility of $A$ matrix. The detailed proof is omitted due to space constraints and can be found in [13].

\section{B. Equilibrium conditions}

We rewrite the equilibrium condition (10) in local form

$$
\begin{gathered}
\Sigma_{[i]}^{D G U}:\left\{\begin{array}{l}
0=\bar{d}_{i} \bar{I}_{i}-I_{\text {load }, i}-\bar{I}_{\text {net }, i} \\
0=-\bar{d}_{i} \bar{V}_{i}-R_{i} \bar{I}_{i}+V_{\text {in }, i}
\end{array}\right. \\
\Sigma_{[j]}^{\text {line }}:\left\{\begin{array}{l}
0=\bar{V}_{\text {net }, j}-R_{L j} \bar{I}_{L j},
\end{array}\right.
\end{gathered}
$$

where the notation - represents the variables at equilibrium. By simplifying (11b), one equivalently obtains

$$
\bar{I}_{L j}=\frac{1}{R_{L j}} \bar{V}_{n e t, j}=\frac{1}{R_{L j}} \sum_{k \in \mathcal{N}_{j}} b_{k j} \bar{V}_{k} .
$$

Then, using (7) and (12), $\bar{I}_{n e t, i}$ can be expressed as

$$
\begin{aligned}
\bar{I}_{n e t, i} & =\sum_{j \in \mathcal{N}_{i}} b_{i j} \frac{1}{R_{L j}} \sum_{k \in \mathcal{N}_{j}} b_{k j} \bar{V}_{k}=\sum_{k \in \mathcal{N}_{j}} \sum_{j \in \mathcal{N}_{i}} \frac{b_{i j} b_{k j}}{R_{L j}} \bar{V}_{k} \\
& =\underbrace{\sum_{j \in \mathcal{N}_{i}} \frac{b_{i j}^{2}}{R_{L j}}}_{1 / \bar{R}_{L i}} \bar{V}_{i}+\underbrace{\sum_{k \in \mathcal{N}_{j}-\{i\}} \sum_{j \in \mathcal{N}_{i}} \frac{b_{i j} b_{k j}}{R_{L j}} \bar{V}_{k}}_{I_{e x t, i}} \\
& =\frac{1}{\bar{R}_{L i}} \bar{V}_{i}+I_{e x t, i} .
\end{aligned}
$$

Formula (13) splits the coupling current into two terms: one proportional to the DGU's own voltage, $\frac{1}{R_{L i}} \bar{V}_{i}$, and another one proportional to the voltages of its neighbouring DGUs, ,$I_{\text {ext }, i}$. Using (12) and (13), and defining $\mathcal{I}_{i}=-\left(I_{\text {load }, i}+\right.$ $\left.I_{\text {ext }, i}\right)$, one can express the unique global equilibrium in a local form as

$$
\begin{aligned}
& \Sigma_{[i]}^{D G U}:\left\{\begin{array}{l}
\bar{V}_{i}=\frac{\bar{R}_{L i} V_{i n, i} \bar{d}_{i}+\bar{R}_{L i} R_{i} \mathcal{I}_{i}}{\bar{R}_{L i} \bar{d}_{i}^{2}+R_{i}} \\
\bar{I}_{i}=\frac{V_{i n, i}-\bar{R}_{L i} \bar{d}_{i} \mathcal{I}_{i}}{\bar{R}_{L i} \bar{d}_{i}^{2}+R_{i}}
\end{array}\right. \\
& \Sigma_{[j]}^{\text {line }}:\left\{\begin{array}{l}
\bar{I}_{L j}=\frac{1}{R_{L j}} \bar{V}_{n e t, j} .
\end{array}\right.
\end{aligned}
$$

It must be noted that the equilibrium PCC voltage $\bar{V}_{i}$ is dependent upon the input voltage $V_{i n, i}$ and duty cycle $d_{i}$. We recall that $d_{i} \in(0,1)$, which leads to a limit on maximum attainable $\bar{V}_{i}$. To deduce this limit, we rewrite (14a) in terms of $\bar{d}_{i}$ and obtain two solutions

$$
\bar{d}_{i, 1}=\frac{\bar{R}_{L i} V_{i n, i}+\sqrt{\Delta_{i}}}{2 \bar{R}_{L i} \bar{V}_{i}} \text { and } \bar{d}_{i, 2}=\frac{\bar{R}_{L i} V_{i n, i}-\sqrt{\Delta_{i}}}{2 \bar{R}_{L i} \bar{V}_{i}},
$$

where $\Delta_{i}=\left(\bar{R}_{L i} V_{i n, i}\right)^{2}-4 \bar{R}_{L i} R_{i} \bar{V}_{i}\left(\bar{V}_{i}-\bar{R}_{L i} \mathcal{I}_{i}\right)$. Notice that duty cycles $\bar{d}_{i, 1}$ and $\bar{d}_{i, 2}$ are the open-loop control inputs able to sustain an output voltage equal to $\bar{V}_{i}$. In order to deduce the maximum attainable equilibrium voltage, we enforce the the limits of $d_{i}$ on $\bar{d}_{i, 1}$, which is the greater of the two solutions. On further simplification, one obtains

$$
V_{i n, i}<\bar{V}_{i}<\frac{1}{2} \bar{R}_{L i} \mathcal{I}_{i}+\sqrt{\left(\frac{1}{2} \bar{R}_{L i} \mathcal{I}_{i}\right)^{2}+\frac{\bar{R}_{L i}}{4 R_{i}} V_{i n, i}^{2}}
$$




\section{DESIGN OF NON-LiNEAR LOCAL REGUlators}

The main control objective is to steer the voltage at $P C C_{i}$ to a reference value $\bar{V}_{i}=V_{\text {ref,i } i}$ at equilibrium, while satisfying constraints (15). Therefore, at equilibrium,

$$
\lim _{t \rightarrow \infty}\left(V_{i}(t)-V_{\text {ref,i }}\right)=0 \quad \forall i \in \mathcal{S}_{A} .
$$

We firstly introduce the following shift of variables

$$
\begin{aligned}
& V_{i}=\bar{V}_{i}+\tilde{V}_{i}, \\
& I_{i}=\bar{I}_{i}+\tilde{I}_{i}, \\
& I_{L j}=\bar{I}_{L j}+\tilde{I}_{L j} .
\end{aligned}
$$

In this way, the new state variables $\tilde{V}_{i}, \tilde{I}_{i}$ and $\tilde{I}_{L j}$ represent a perturbation from the respective equilibria $\bar{V}_{i}, \bar{I}_{i}$ and $\bar{I}_{L j}$. We consider the following non-linear and dynamic control laws [16]

$$
\begin{gathered}
d_{i}=\tilde{d}_{i}=\operatorname{sat}\left(\bar{d}_{i}^{*}+\tilde{\phi}_{i}\right), \\
\dot{\tilde{\phi}}_{i}=\gamma_{i}\left(V_{r e f, i} \tilde{I}_{i}-\bar{I}_{i} \tilde{V}_{i}\right)-\gamma_{i} K_{W i}\left(\tilde{d}_{i}-\bar{d}_{i}^{*}\right),
\end{gathered}
$$

where $\gamma_{i}, K_{W i}$ are positive constants, $\tilde{\phi}_{i}$ is a new state variable and $\bar{d}_{i}^{*}=\left.\bar{d}_{i, 1}\right|_{\bar{V}_{i}=V_{r e f, i}}$. The function $\operatorname{sat}(\cdot)$ is defined as

$$
\operatorname{sat}(\cdot)=\left\{\begin{array}{l}
d_{\max } \text { if }(\cdot) \geq d_{\max } \\
(\cdot) \text { if } d_{\min }<(\cdot)<d_{\max } \\
d_{\min } \text { if }(\cdot) \leq d_{\min }
\end{array}\right.
$$

where $0<d_{\min }<\bar{d}_{i}^{*}<d_{\max }<1$. By operating the shift (17), and substituting controllers (18) into systems (4)-(6), the resulting shifted closed-loop system is

$$
\begin{aligned}
& \tilde{\Sigma}_{[i]}^{D G U}: \\
& \left\{\begin{array}{c}
C_{i} \dot{\tilde{V}}_{i}=\tilde{d}_{i}\left(\bar{I}_{i}+\tilde{I}_{i}\right)-I_{\text {load }, i}-\left(\bar{I}_{n e t, i}+\tilde{I}_{n e t, i}\right) \\
L_{i} \dot{\tilde{I}}_{i}=-\tilde{d}_{i}\left(V_{\text {ref }, i}+\tilde{V}_{i}\right)-R_{i}\left(\bar{I}_{i}+\tilde{I}_{i}\right)+V_{i n, i} \\
\dot{\tilde{\phi}}_{i}=\gamma_{i}\left(V_{\text {ref }, i} \tilde{I}_{i}-\bar{I}_{i} \tilde{V}_{i}\right)-\gamma_{i} K_{W i}\left(\tilde{d}_{i}-\bar{d}_{i}^{*}\right)
\end{array}\right. \\
& \tilde{\Sigma}_{[j]}^{l i n e}: \\
& \left\{\begin{array}{l}
L_{L j} \dot{\tilde{I}}_{L j}=\left(\bar{V}_{n e t, j}+\tilde{V}_{n e t, j}\right)-R_{L j}\left(\bar{I}_{L j}+\tilde{I}_{L j}\right) .
\end{array}\right.
\end{aligned}
$$

Equivalently, (20) can be represented in control-affine form (1) as

$$
\begin{aligned}
& \tilde{\Sigma}_{[i]}^{D G U}:\left\{\begin{array}{l}
\dot{\tilde{x}}_{[i]}=q_{[i]}\left(\tilde{x}_{[i]}, \tilde{u}_{[i]}\right)=\tilde{f}_{[i]}\left(\tilde{x}_{[i]}\right)+\tilde{g}_{[i]}\left(\tilde{x}_{[i]}\right) \tilde{u}_{[i]} \\
\tilde{y}_{[i]}=\tilde{h}_{[i]}\left(\tilde{x}_{[i]}\right)
\end{array}\right. \\
& \tilde{\Sigma}_{[j]}^{l i n e}:\left\{\begin{array}{l}
\dot{\tilde{x}}_{[j]}=q_{[j]}\left(\tilde{x}_{[j]}, \tilde{u}_{[j]}\right)=\tilde{f}_{[j]}\left(\tilde{x}_{[j]}\right)+\tilde{g}_{[j]}\left(\tilde{x}_{[j]}\right) \tilde{u}_{[j]} \\
\tilde{y}_{[j]}=\tilde{h}_{[j]}\left(\tilde{x}_{[j]}\right)
\end{array},\right.
\end{aligned}
$$

where $i \in \mathcal{S}_{A}, j \in \mathcal{S}_{L}, \tilde{x}_{[i]}=\left[\begin{array}{lll}\tilde{V}_{i} & \tilde{I}_{i} & \tilde{\phi}_{i}\end{array}\right]^{T} \in \mathbb{R}^{3}, \tilde{y}_{[i]}=\tilde{V}_{i}$, and

$$
\tilde{u}_{[i]}=-\tilde{I}_{n e t, i}=-\sum_{j \in \mathcal{N}_{i}} b_{i j} \tilde{I}_{L j} .
$$

Similarly, $\tilde{x}_{[j]}=\tilde{I}_{L j}, \tilde{y}_{[j]}=\tilde{I}_{L j}$, and

$$
\tilde{u}_{[j]}=\tilde{V}_{n e t, j}=\sum_{k \in \mathcal{N}_{j}} b_{k j} \tilde{V}_{k} .
$$

Remark 1: It can be shown that $q_{[i]}(0,0)=0$ and $q_{[j]}(0,0)=0$. Therefore, $\tilde{x}_{[i]}=0$ and $\tilde{x}_{[j]}=0$ are the respective equilibrium states of $\tilde{\Sigma}_{[i]}^{D G U}$ and $\tilde{\Sigma}_{[j]}^{\text {Line }}$.

Moreover, we want to point out that the control law $d_{i}$ explicitly takes into account the saturation of the duty cycle. Furthermore, we introduce two design parameters, $d_{\text {min }}$ and $d_{\max }$, that can be selected to arbitrarily limit the saturation width. In order to design regulators (18), the following parameters have to be determined:

- $\gamma_{i}$ and $K_{W i}$, which can be freely chosen to influence $\phi_{i}$ dynamics;

- $d_{\min }, d_{\max } \in(0,1)$;

- $V_{\text {ref,i }}$, which must be selected satisfying constraint (15);

- $\bar{d}_{i}^{*}$, which in turn depends on $i$-th DGU's electrical parameters, on neighboring line resistances $R_{L j}$ and on 2-hop neighbors (i.e. neighboring DGUs) voltage references $V_{\text {ref, }, k}$;

- $\bar{I}_{i}$, which requires the same parameters mentioned above.

Hence, regulators (18) are local in the sense that they only need DGU's electrical parameters, in addition to the voltage references of its neighboring DGUs and resistances of the neighboring lines. Therefore, when a DGU is plugged -in or -out to the $\mathrm{mG}$, only regulators of DGUs whose set of neighbors has changed must be retuned. Conversely, all other controllers remain unchanged.

\section{Passivity of Closed-Loop DGUs}

The scope of this section is to prove that passivity holds for closed-loop DGU models. We know that RL lines are strictly passive with respect to input $\tilde{u}_{[j]}=\tilde{V}_{n e t, j}$ and output $\tilde{y}_{[j]}=\tilde{I}_{L j}$. In fact, as shown in [2], by introducing a positive definite and radially unbounded storage function of the form

$$
\mathcal{V}_{L, j}\left(\tilde{I}_{L j}\right)=\frac{1}{2} L_{L j} \tilde{I}_{L j}^{2},
$$

the following passivity condition holds

$$
\tilde{V}_{n e t, j} \tilde{I}_{L j}=\dot{\mathcal{V}}_{L, j}+\underbrace{R_{L j} \tilde{I}_{L j}^{2}}_{\psi_{L, j}\left(\tilde{I}_{L j}\right) \geq 0} .
$$

Passivity of RL lines, together with the passivity of DGUs, allows us to prove voltage stability in the whole $\mathrm{mG}$ (see Section VI). To this aim, we firstly introduce the following result [16].

Proposition 1: The function

$$
\mathcal{U}_{i}\left(\tilde{\phi}_{i}\right)=\frac{\tilde{\phi}_{i}^{2}}{2}-\int_{0}^{\tilde{\phi}_{i}}\left(\bar{d}_{i}^{*}+\tilde{\phi}_{i}-\tilde{d}_{i}\right) d \tilde{\phi}_{i}
$$

is positive definite.

Now we are able to state the main result.

Proposition 2: The DGU (20a) is passive with respect to input $\tilde{u}_{[i]}=-\tilde{I}_{n e t, i}$ and output $\tilde{y}_{[i]}=\tilde{V}_{i}$.

Proof: Consider the following positive definite and radially unbounded storage function

$$
\mathcal{V}_{A, i}\left(\tilde{V}_{i}, \tilde{I}_{i}, \tilde{\phi}_{i}\right)=\frac{1}{2} C_{i} \tilde{V}_{i}^{2}+\frac{1}{2} L_{i} \tilde{I}_{i}^{2}+\gamma_{i}^{-1} \mathcal{U}_{i}\left(\tilde{\phi}_{i}\right)
$$


On evaluating its derivative along the trajectories of (20a), one obtains

$$
\begin{aligned}
\dot{\mathcal{V}}_{A, i}= & C_{i} \tilde{V}_{i} \dot{\tilde{V}}_{i}+L_{i} \tilde{I}_{i} \dot{\tilde{I}}_{i}+\gamma_{i}^{-1}\left(\tilde{\phi}_{i}-\left(\bar{d}_{i}^{*}+\tilde{\phi}_{i}-\tilde{d}_{i}\right)\right) \dot{\tilde{\phi}}_{i} \\
= & (\underbrace{\left(\bar{d}_{i}^{*} \bar{I}_{i}-I_{\text {load }, i}+\bar{I}_{n e t, i}\right.}_{a=0}) \tilde{V}_{i}+(\underbrace{V_{i n, i}-R_{i} \bar{I}_{i}-\bar{d}_{i}^{*} \bar{V}_{i}}_{b=0}) \tilde{I}_{i} \\
& -\tilde{I}_{n e t, i} \tilde{V}_{i}-R_{i} \tilde{I}_{i}^{2}-K_{W i}\left(\tilde{d}_{i}-\bar{d}_{i}^{*}\right)^{2} .
\end{aligned}
$$

Notice that terms $a$ and $b$ represent equilibrium conditions (11a), and therefore they are equal to zero. Equivalently, we can write

$$
\left(-\tilde{I}_{n e t, i}\right) \tilde{V}_{i}=\dot{\mathcal{V}}_{A, i}+\underbrace{R_{i} \tilde{I}_{i}^{2}+K_{W i}\left(\tilde{d}_{i}-\bar{d}_{i}^{*}\right)^{2}}_{\psi_{A, i}\left(\tilde{V}_{i}, \tilde{I}_{i}, \tilde{\phi}_{i}\right) \geq 0} .
$$

Hence, system (20a) is passive with respect to input $-\tilde{I}_{n e t, i}$ and output $\tilde{V}_{i}$.

\section{Network Global Asymptotic Stability}

In this following Theorem, we exploit the passivity properties of DGUs and lines to prove global asymptotic stability of the origin of the system $\Sigma$ given by (21), (22), and (23). In particular, the proof of the Theorem utilizes results in [17] for characterizing stability properties of passive systems interconnected in a skew symmetric fashion.

Theorem 1 (Stability of the microgrid): The origin of the system $\Sigma$ given by (21), (22), and (23) is globally asymptotically stable.

Proof: DGUs (20a) are passive with input $\tilde{u}_{[i]}=$ $-\tilde{I}_{n e t, i}$ and output $\tilde{y}_{[i]}=\tilde{V}_{i}, i \in \mathcal{S}_{A}$. Power lines (20b) are passive with respect to input $\tilde{u}_{[j]}=\tilde{V}_{n e t, j}$ and output $\tilde{y}_{[j]}=\tilde{I}_{l, j}, j \in \mathcal{S}_{L}$. It is shown in [17, Lemma 1], $\tilde{u}_{[i]}$ and $\tilde{u}_{[j]}$ correspond to the so-called skew-symmetric interconnections. Moreover, the lines and DGUs not only have control-affine dynamics (see (21)), but also are passive with radially unbounded functions. This is sufficient to invoke Corollary 1 in [17], which guarantees that the state $\tilde{x}=\left[\tilde{x}_{[1]}, \cdots, \tilde{x}_{[p+q]}\right]^{T}$ converges, as $t \rightarrow \infty$, to the largest invariant set contained in

$$
\begin{array}{r}
E=\left\{\tilde{x}_{[i]}, \tilde{x}_{[j]}: \psi_{A, i}\left(\tilde{x}_{[i]}\right)=0, \psi_{L, j}\left(\tilde{x}_{[j]}\right)=0,\right. \\
\left.i \in \mathcal{S}_{A}, j \in \mathcal{S}_{L}\right\} .
\end{array}
$$

In order to characterize set $E$, we impose

$$
\left\{\begin{array}{l}
\psi_{A, i}\left(\tilde{x}_{[i]}\right)=R_{i} \tilde{I}_{i}^{2}+K_{W, i}\left(\tilde{d}_{i}-\bar{d}_{i}^{*}\right)^{2}=0 \\
\psi_{L, j}\left(\tilde{x}_{[j]}\right)=R_{L j} \tilde{I}_{L j}^{2}=0
\end{array},\right.
$$

which is satisfied by the family of vectors of the form

$$
\left\{\begin{array}{l}
\tilde{x}_{[i]}=\left[\begin{array}{c}
\tilde{V}_{i} \\
\tilde{I}_{i} \\
\tilde{\phi}_{i}
\end{array}\right]=\left[\begin{array}{c}
\alpha_{i} \\
0 \\
0
\end{array}\right], \quad i \in \mathcal{S}_{A}, j \in \mathcal{S}_{L} . \\
\tilde{x}_{[j]}=\tilde{I}_{L j}=0
\end{array}\right.
$$

In order to find the largest invariant set $M \subseteq E$, we aim to deduce conditions on $\tilde{x}_{[i]}(0), \tilde{x}_{[j]}(0) \in E$, such that $\dot{\tilde{x}}_{[i]}(0), \dot{\tilde{x}}_{[j]}(0) \in E$. Using (21), one has

$$
\begin{aligned}
& \dot{\tilde{x}}_{[i]}(0)=\tilde{f}_{[i]}\left(\tilde{x}_{[i]}(0)\right)+\left.\tilde{g}_{[i]}\left(\tilde{x}_{[i]}(0)\right)\left(-\tilde{I}_{n e t, i}\right)\right|_{\tilde{I}_{L j}=0} \\
& =\left[\begin{array}{c}
\frac{1}{C_{i}}\left(\bar{d}_{i}^{*} \bar{I}_{i}-I_{\text {load }, i}-\bar{I}_{\text {net }, i}-\left.\tilde{I}_{n e t, i}\right|_{\tilde{I}_{L j}=0}\right) \\
\overline{-1}\left(\bar{d}_{i}^{*}\left(\bar{V}_{i}+\alpha_{i}\right)+R_{i} \bar{I}_{i}+V_{i n, i}\right) \\
-\gamma_{i} \bar{I}_{i} \alpha_{i}-\gamma_{i} K_{W i}\left(\operatorname{sat}\left(\bar{d}_{i}^{*}\right)-\bar{d}_{i}^{*}\right)
\end{array}\right] \\
& =\left[\begin{array}{c}
0 \\
-\frac{\bar{d}_{i}^{*} \alpha_{i}}{L_{i}} \\
-\gamma_{i} \bar{I}_{i} \alpha_{i}-\gamma_{i} K_{W i}\left(\operatorname{sat}\left(\bar{d}_{i}^{*}\right)-\bar{d}_{i}^{*}\right)
\end{array}\right], \\
& \dot{\tilde{x}}_{[j]}(0)=\tilde{f}_{[j]}\left(\tilde{x}_{[j]}(0)\right)+\left.\tilde{g}_{[j]}\left(\tilde{x}_{[j]}(0)\right) \tilde{V}_{n e t, j}\right|_{\tilde{V}_{i}=\alpha_{i}} \\
& =\bar{V}_{n e t, j}+\left.\tilde{V}_{n e t, j}\right|_{\tilde{V}_{i}=\alpha_{i}}-R_{L j}\left(\bar{I}_{L j}+\tilde{I}_{L j}\right)=0 .
\end{aligned}
$$

We, now, impose (32) to be confined in the set $E$ at all instants in the future. From (31), we obtain the following set of linear equations in $\alpha_{i}$

$$
\left\{\begin{array}{l}
0=\alpha_{i} \\
\bar{d}_{i}^{*} \alpha_{i}=0 \\
\gamma_{i} \bar{I}_{i} \alpha_{i}+\gamma_{i} K_{W i}\left(\operatorname{sat}\left(\bar{d}_{i}^{*}\right)-\bar{d}_{i}^{*}\right)=0
\end{array}\right.
$$

whose unique solution is

$$
\alpha_{i}=0, \quad \forall i \in \mathcal{S}_{A} .
$$

Hence, the largest invariant set $M \subseteq E$ is the origin of the state space. This means that the origin of system $\Sigma$ is globally asymptotically stable.

As a consequence of Theorem 1, controllers (18) are able to stabilize the global dynamics and steer each PCC voltage to its reference value (recall that $\bar{V}_{i}=V_{\text {ref,i }} \forall i \in \mathcal{S}_{A}$ ).

\section{Simulation Results}

In this section, the stabilization properties of the proposed control architecture are evaluated through simulations, which are conducted using Simulink Power Systems toolbox. We consider a set of 5 identical DGUs depicted in Figure $3(I)$. Each node embeds a closed-loop DGU along with a local load. Connecting lines consist of a resistance $R=1 \Omega$, in series with an inductance $L=1 \mu H$. At the beginning of the simulation, each DGU is disconnected from the $\mathrm{mG}$ (see Figure $3(I))$ and is steered to a reference voltage $V_{\text {ref, } i}$. The electrical and control parameters of the DGUs along with reference voltages are reported in Table I. Then, a sequence of plug-in operations, depicted in Figure $3(I I)$ and $(I I I)$, are conducted. The control objective is to guarantee that each DGU tracks its reference value, even when the couplings are modified. At times $T_{1}=1 \mathrm{~s}$ and $T_{2}=1.5 \mathrm{~s}$, when the $\mathrm{mG}$ couplings are modified, the regulators of DGUs whose set of neighbours have changed must be retuned (see Section IV), while all other controllers remain unchanged. For instance, at time $T_{2}$, regulators of DGUs 2, 3 and 5 are updated. Retuning operation simply requires re-computing the term $\bar{d}_{i}^{*}$ (defined in Section III-B), taking into consideration the reference values of the new set of neighbors. As observed in Figure 4, the decentralized architecture is able to asymptotically track each reference signal $V_{r e f, i}$, in the presence of the electrical 
couplings. We observe voltage transients in proximity of the plug-in times $T_{1}$ and $T_{2}$, but the voltages are restored to the reference values.

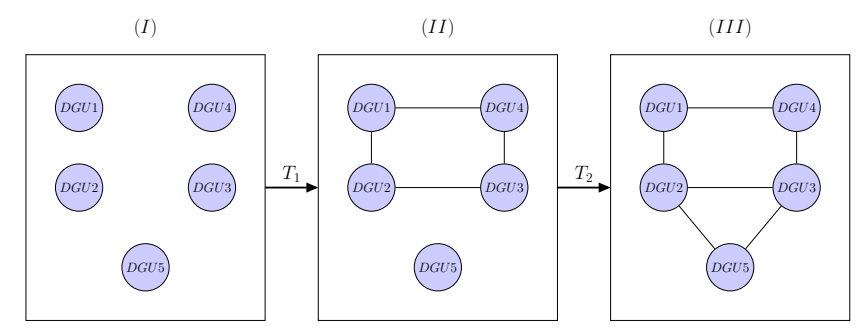

Fig. 3: Sequence of plug-in operations $\left(T_{1}=1 s, T_{2}=\right.$ $1.5 s)$.
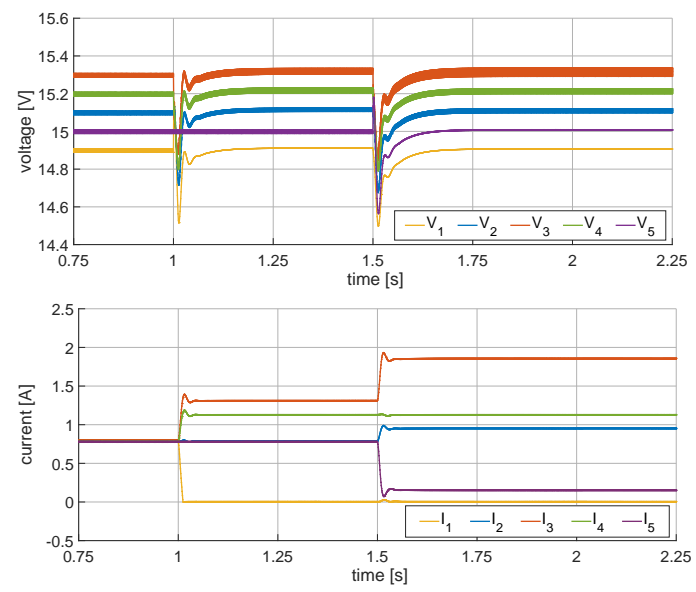

Fig. 4: Control simulation of an islanded MVDC $\mathrm{mG}$.

\begin{tabular}{|c|c|}
\hline \multicolumn{2}{|c|}{ Electrical parameters } \\
\hline$V_{i n}$ & $10 \mathrm{~V}$ \\
$R_{c}$ & $10 \Omega$ \\
$C$ & $1 \mathrm{mF}$ \\
$L$ & $50 \mathrm{mH}$ \\
$R$ & $0.5 \Omega$ \\
$f$ & $1 \mathrm{kHz}$ \\
\hline \multicolumn{2}{|c|}{ Control parameters } \\
\hline$d_{\min }$ & 0.1 \\
$d_{\max }$ & 0.9 \\
$K_{W}$ & 10 \\
$\gamma$ & 20 \\
\hline
\end{tabular}

\begin{tabular}{|c|c|}
\hline \multicolumn{2}{|c|}{ Reference values (in Volts) } \\
\hline$V_{\text {ref }, 1}$ & 14.9 \\
$V_{\text {ref }, 2}$ & 15.1 \\
$V_{\text {ref }, 3}$ & 15.3 \\
$V_{\text {ref }, 4}$ & 15.2 \\
$V_{\text {ref }, 5}$ & 15 \\
\hline
\end{tabular}

TABLE I: Simulation parameters

\section{CONCLUSIONS}

In this work, we dealt with the problem of voltage stability in MVDC mGs. An $\mathrm{mG}$ model based on nonlinear DGUs interconnected by power lines was considered. Utilizing a passivity-based framework, we proposed a decentralized nonlinear primary control architecture, which allows PnP operations. Design of regulators can be performed explicitly, relying on local electrical quantities and voltage references. Our PnP control architecture guarantees global asymptotic stability of the $\mathrm{mG}$.

A further analysis on evaluation of control performances is deferred for future work. Moreover, a major limitation is the absence of an integral action in local controllers (18). This presumes a perfect knowledge of the DGUs electrical parameters, without which the zero-error reference tracking cannot be guaranteed. Future developments will focus on extending MV PnP regulators in this direction.

\section{REFERENCES}

[1] M. Andreasson, R. Wiget, D. V. Dimarogonas, K. H. Johansson, and G. Andersson, "Distributed frequency control through MTDC transmission systems," IEEE Transactions on Power Systems, vol. 32, no. 1, pp. 250-260, 2017.

[2] B. Brogliato, R. Lozano, B. Maschke, and O. Egeland, Dissipative systems analysis and control. Springer, London, 2007.

[3] M. Burger and C. D. Persis, "Dynamic coupling design for nonlinear output agreement and time-varying flow control," Automatica, vol. 51, pp. $210-222,2015$.

[4] N. Chopra, "Output synchronization on strongly connected graphs," IEEE Transactions on Automatic Control, vol. 57, no. 11, pp. 28962901, 2012.

[5] T. Dragičević, X. Lu, J. C. Vasquez, and J. M. Guerrero, "DC microgrids part I: A review of control strategies and stabilization techniques," IEEE Transactions on Power Electronics, vol. 31, no. 5, pp. 4876-4891, 2016.

[6] — "DC microgrids part II: A review of power architectures, applications, and standardization issues," IEEE Transactions on Power Electronics, vol. 31, no. 5, pp. 3528-3549, 2016.

[7] M. Farina, A. Guagliardi, F. Mariani, C. Sandroni, and R. Scattolini, "Model predictive control of voltage profiles in MV networks with distributed generation," Control Engineering Practice, vol. 34, pp. 18 $-29,2015$.

[8] N. Flourentzou, V. G. Agelidis, and G. D. Demetriades, "VSC-based HVDC power transmission systems: An overview," IEEE Transactions on Power Electronics, vol. 24, no. 3, pp. 592-602, 2009.

[9] J. M. Guerrero, J. C. Vasquez, J. Matas, D. Vicuna, L. Garcia, and M. Castilla, "Hierarchical control of droop-controlled AC and DC microgrids - a general approach toward standardization," IEEE Transactions on Industrial Electronics, vol. 58, no. 1, pp. 158-172, 2011.

[10] H. K. Khalil, Nonlinear Systems, 3rd ed. Prentice Hall, New Jersey, 2002.

[11] N. Kottenstette, M. J. McCourt, M. Xia, V. Gupta, and P. J. Antsaklis, "On relationships among passivity, positive realness, and dissipativity in linear systems," Automatica, vol. 50, no. 4, pp. 1003-1016, 2014.

[12] R. Lasseter, A. Akhil, C. Marnay, J. Stephens, J. Dagle, R. Guttromson, A. Meliopoulous, R. Yinger, , and J. Eto, "The CERTS microgrid concept," Office of Power Technologies, US Department of Energy, Tech. Rep., 2002, white Paper for Transmission Reliability Program.

[13] A. Martinelli, "A passivity-based approach to voltage stabilization in DC microgrids," Master's thesis, Politechnico Milano, 2017.

[14] L. Meng, Q. Shafiee, G. Ferrari-Trecate, H. Karimi, D. Fulwani, X. Lu, and J. M. Guerrero, "Review on control of DC microgrids and multiple microgrid clusters," IEEE Journal of Emerging and Selected Topics in Power Electronics, vol. 5, no. 3, pp. 928-948, 2017.

[15] R. D. Middlebrook and S. Cuk, "A general unified approach to modelling switching-converter power stages," in 1976 IEEE Power Electronics Specialists Conference, June 1976, pp. 18-34.

[16] J. Moreno-Valanzuela and O. Garcia-Alarcon, "On control of a boost DC-DC converter under constrained input," Complexity, vol. 2017, p. 11, 2017.

[17] P. Nahata, R. Soloperto, M. Tucci, A. Martinelli, and G. FerrariTrecate, "A passivity-based approach to voltage stabilization in DC microgrids with ZIP loads," Automatica, 2018, submitted. Available online at https://infoscience.epfl.ch/record/253266.

[18] M. Tucci, S. Riverso, and G. Ferrari-Trecate, "Line-independent plug-and-play controllers for voltage stabilization in DC microgrids," IEEE Transactions on Control Systems Technology, 2018, to appear. DOI=10.1109/TCST.2017.2695167.

[19] M. Tucci, S. Riverso, J. C. Vasquez, J. M. Guerrero, and G. F. Trecate, "A decentralized scalable approach to voltage control of DC islanded microgrids," IEEE Transactions on Control Systems Technology, vol. 24, no. 6, pp. 1965-1979, 2016.

[20] J. Zhao and F. Dorfler, "Distributed control and optimization in DC microgrids," Automatica, vol. 61, pp. 18-26, 2015.

[21] D. Zonetti, R. Ortega, and J. Schiffer, "A tool for stability and power sharing analysis of a generalized class of droop controllers for highvoltage direct-current transmission systems," IEEE Transactions on Control of Network Systems, 2017. 